\title{
Evaluation of Alitretinoin for the Treatment of Mycosis Fungoides and Sézary Syndrome
}

\author{
Raed Alhusayen ${ }^{a, b}$ Trang Tuyet $\mathrm{Vu}^{\mathrm{c}}$ Nouf Almuhanna ${ }^{\mathrm{a}, \mathrm{b}, \mathrm{d}}$ \\ Iris Wohlmuth-Wieser ${ }^{a, b}$, e Jori Hardin ${ }^{f}$ John-Douglas Matthew Hughes ${ }^{f}$ \\ Justin Chia ${ }^{f}$ Leslie Street ${ }^{g}$ Neil Hartley Shear ${ }^{a}$ Scott Richard Walsh ${ }^{a}$ b \\ Robert Gniadeckic, h

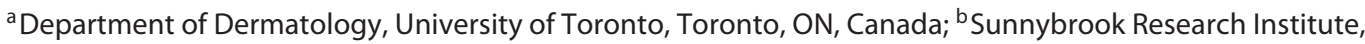 \\ University of Toronto, Toronto, ON, Canada; ' ${ }^{C}$ Department of Dermatology, University of Alberta, \\ Edmonton, AB, Canada; ${ }^{\mathrm{d} D e p a r t m e n t}$ of Dermatology, King Fahad Medical City, Riyadh, Saudi Arabia; ${ }^{e}$ Department \\ of Dermatology, Paracelsus Medical University, Salzburg, Austria; ${ }^{\mathrm{D}}$ Department of Dermatology, University of \\ Calgary, Calgary, AB, Canada; 9 Department of Hematology-Oncology, University of Calgary, Calgary, AB, Canada; \\ ${ }^{\mathrm{h}}$ Department of Dermatology, University of Copenhagen, Copenhagen, Denmark
}

\section{Keywords}

Alitretinoin - Mycosis fungoides - Sézary syndrome .

Cutaneous T-cell lymphoma $\cdot$ Retrospective study

\section{Abstract}

Background: Mycosis fungoides (MF) and Sézary syndrome (SS) are the most common subtypes of cutaneous T-cell lymphoma (CTCL). There is currently no cure for $\mathrm{CTCL}$, and treatment is aimed at limiting disease progression. This study evaluated the efficacy and tolerability of alitretinoin in CTCL management. Methods: A retrospective, multicenter study was conducted on CTCL patients treated with alitretinoin as a primary agent or in combination with standard therapies. Results: Forty-eight patients with MF $(n=40)$ and SS $(n=8)$ with a median age of 59.7 years $( \pm 14.3)$ were eligible for study inclusion. Treatment response data were evaluated in 40 patients and safety in 42 patients. $40.0 \%$ of the patients had early-stage, $43.8 \%$ had advanced-stage $\mathrm{CTCL}$, and in $16.7 \%$ of patients there was insufficient information for staging. $40.0 \%(16 / 40)$ of the patients achieved a complete or partial response, whereas $47.5 \%$ (19/40) achieved stable dis-

karger@karger.com www.karger.com/drm

Karger ${ }^{\prime \prime} \div$

GOPEN ACCESS
(C) 2021 The Author(s)

Published by S. Karger AG, Basel

This is an Open Access article licensed under the Creative Commons Attribution-NonCommercial-4.0 International License (CC BY-NC) (http://www.karger.com/Services/OpenAccessLicense), applicable to the online version of the article only. Usage and distribution for commercial purposes requires written permission. ease, 12.5\% (5/40) had progressive disease, and there were no cases of disease relapses in responders. Both early and advanced stages of $C T C L$ were responsive to alitretinoin as a primary or combined modality. Alitretinoin was well tolerated, and $64.3 \%$ (27/42) of patients did not report any side effects. The most commonly observed side effect was hypertriglyceridemia. Conclusions: This retrospective analysis supports the efficacy and safety of alitretinoin in clearing skin disease and preventing disease progression in CTCL as a monotherapy or in combination with standard therapies.

(c) 2021 The Author(s)
Published by S. Karger AG, Basel

\section{Introduction}

Cutaneous T-cell lymphoma (CTCL) is a heterogeneous group of non-Hodgkin's lymphomas characterized by skin-limited infiltration of neoplastic $\mathrm{T}$ lymphocytes. Mycosis fungoides (MF) is the most prevalent type of CTCL, accounting for up to $65 \%$ of cases [1]. In MF, the disease course is indolent and mostly skin limited, with patch or plaque stage disease that can persist for years to 
Fig. 1. Flow chart of the methods: overview of study procedure. MF, mycosis fungoides; SS, Sézary syndrome; ISCL, International Society of Cutaneous Lymphoma.

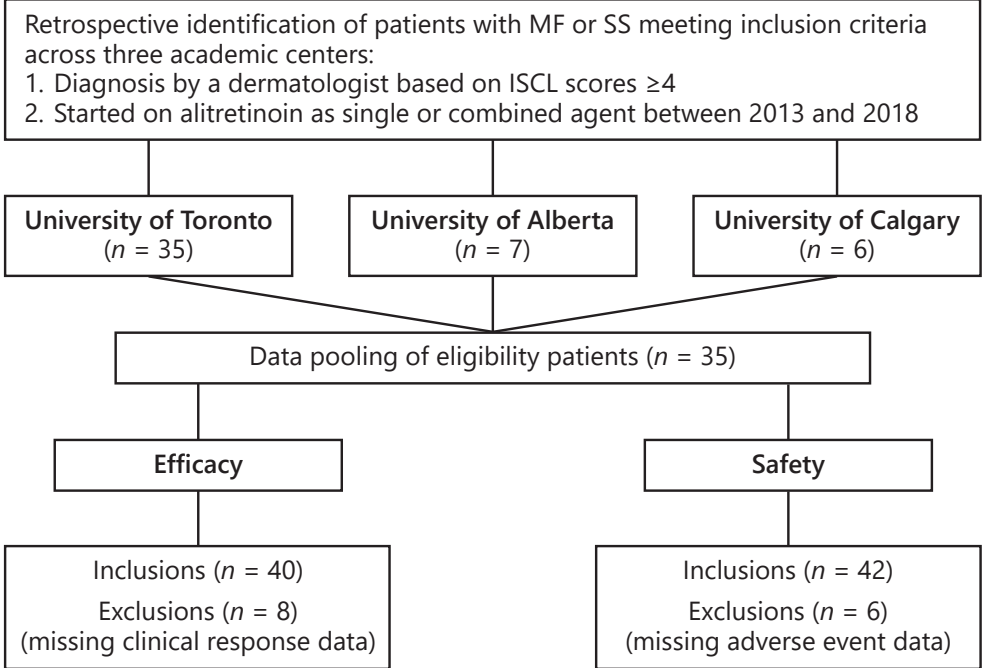

Retrospective identification of patients with MF or SS meeting inclusion criteria

. Diagnosis by a dermatologist based on ISCL scores $\geq 4$ decades [2]. Skin-limited MF can evolve through the patch, plaque and tumor stages, and can infiltrate the lymph nodes, viscera and blood [2]. The overall 5-year survival for patch/plaque stage IB disease is $86 \%$ with worsening mortality upon upstaging. Systemic involvement portends worse prognosis, with the overall 5-year survival for nodal, visceral and organ involvement in stage IVB disease being 23\% [3]. Sézary syndrome (SS) is a more aggressive subtype than MF and is classically viewed as a leukemic form of CTCL presenting with erythroderma [1].

No curative therapies currently exist for MF and SS. The goal of treatment is to mitigate disease progression and provide symptom control. Management of CTCL depends on the disease stage $[1,2]$. In early skin-limited patch and plaque stage MF (stage IA, IB), topical treatments and phototherapy are the mainstay. In patients with widely distributed or recalcitrant disease, retinoids are one of the first-line systemic options $[2,4]$.

Retinoids are synthetic derivatives of vitamin A and are small, lipophilic molecules that bind to retinoic acid receptors (RARs) and/or retinoid X receptors (RXRs) in the nucleus [5]. The activated retinoid receptors modulate gene transcription in cellular pathways involved in immune regulation, cell proliferation and differentiation [6]. Isotretinoin and acitretin bind RARs, whereas bexarotene binds specifically to RXR, and alitretinoin binds both RARs and RXRs. Of the retinoids, only bexarotene is currently approved for the treatment of CTCL $[6,7]$. In
Table 1. Patient demographics

$\begin{array}{lc}\text { Mean age at diagnosis } \pm \text { SD, years } & 59.7 \pm 14.3 \\ \text { Sex, } n / \text { total }(\%) & \\ \text { Male } & 30 / 48(62.5) \\ \text { Female } & 18 / 48(37.5) \\ \text { TNMB stage MF/SS, } n \text { /total (\%) } & \\ \text { MF IA } & 9 / 48(18.8) \\ \text { MF IB } & 10 / 48(20.8) \\ \text { MF IIA } & 0 / 48(0) \\ \text { MF IIB } & 8 / 48(16.7) \\ \text { MF IIIA } & 2 / 48(4.2) \\ \text { MF IIIB } & 1 / 48(2.1) \\ \text { MF IVA } & 2 / 48(4.2) \\ \text { MF IVB } & 0 / 48(0) \\ \text { SS } & 8 / 48(16.7) \\ \text { Unknown } & 8 / 48(16.7)\end{array}$

MF, mycosis fungoides; TNMB, tumor-node-metastasisblood; SS, Sézary syndrome.

phase 2 and 3 clinical trials, bexarotene was effective in treating approximately $50 \%$ of patients with refractory or persistent early-stage of CTCL, and long-term retrospective studies have shown similar outcomes $[8,9]$. However, bexarotene has a high frequency of adverse events and is not readily accessible in some countries such as Canada $[8,9]$.

A small, retrospective study of MF and SS treated with alitretinoin showed an improvement in skin disease with 
Table 2. Concomitant treatment with alitretinoin

\begin{tabular}{lr}
\hline Median total therapies with alitretinoin (range) & $2(0-5)$ \\
Common combinations with alitretinoin, $n /$ total (\%) & \\
Single-agent alitretinoin & $5 / 48(10.4)$ \\
Topical corticosteroids & $16 / 48(33.3)$ \\
Topical corticosteroid and NBUVB & $6 / 48(12.5)$ \\
Topical corticosteroids and local radiation & $1 / 48(2.1)$ \\
Topical corticosteroids and local radiation and phototherapy & $3 / 48(6.3)$ \\
Topical corticosteroid and prednisone & $2 / 48(4.2)$ \\
Other combinations & $15 / 48(31.3)$ \\
\hline
\end{tabular}

NBUVB, narrow-band ultraviolet light B. minimal side effects [10]. Larger studies are needed to determine whether alitretinoin is an effective and safe treatment option for MF and SS. We report a 5-year, nationwide retrospective review of 48 patients with MF and SS treated with alitretinoin monotherapy or in combination with other standard therapies across 3 academic centers in Canada.

\section{Methods}

For further details, see the online supplementary material (see www.karger.com/doi/10.1159/000512484) (Fig. 1) [10, 11].

\section{Results}

\section{Study Population}

A retrospective chart review was conducted across 3 academic centers in Canada of 48 patients with MF $(n=$ $40)$ and SS $(n=8)$ treated with oral alitretinoin (10-30 mg daily) monotherapy or in combination with standard therapies. Of the 48 patients screened, 40 and 42 patients had complete charts for analyses of treatment response and adverse events, respectively.

\section{Patient Demographics}

Table 1 summarizes the patient demographics. The mean age of diagnosis was $59.7 \pm 14.3$ years (range $24-89$ years). The gender distribution was $62.5 \%$ males compared with $37.5 \%$ females. 39.6\% (19/48) of patients had early-stage CTCL (IA: $18.8 \%$, IB: $20.8 \%$ ), whereas $27.1 \%$ $(13 / 48)$ had advanced-stage disease (IIB: $16.7 \%$, IIIA: $4.2 \%$, IIIB: $2.1 \%$, IVA: $4.2 \%$ ), and $16.7 \%$ (8/48) of patients had SS. $16.7 \%(8 / 48)$ of the patients did not have sufficient information for staging.

Alitretinoin for the Treatment of Mycosis

Fungoides and Sézary Syndrome
Table 3. Response to alitretinoin ( $n /$ total, \%)
Complete response

Partial response

Stable disease

Progressive disease

Disease relapse

Overall response

\author{
$5 / 40(12.5)$ \\ $11 / 40(27.5)$ \\ $19 / 40(47.5)$ \\ $5 / 40(12.5)$ \\ $0 / 40(0)$ \\ $16 / 40(40)$
}

Disease relapse is the number of patients whose skin disease relapsed after obtaining a complete or partial response. Overall response is a composite measure of the complete and partial responses.

\section{Treatment Modalities}

The mean disease duration before the initiation of alitretinoin was 3 years (range $0.1-17$ years). Patients received a median of 3 (range $0-9$ ) treatment modalities prior to starting alitretinoin. While $10.4 \%(5 / 48)$ of patients received alitretinoin as a single agent, $89.6 \%$ (43/48) received alitretinoin in combination with other therapies (Table 2). The median number of concomitant treatments with alitretinoin was 2 (range 0-5). Adjunctive topical corticosteroids were most commonly prescribed with alitretinoin accounting for 33.3\% (16/48), followed by adjunctive topical corticosteroids and narrow-band ultraviolet $\mathrm{B}$ radiation with alitretinoin accounting for $12.5 \%(6 / 48) .31 .3 \%(15 / 48)$ of patients received alitretinoin with various combinations of interferon, methotrexate, extracorporeal electrophoresis, intralesional kenalog and/or other medical modalities (Table 2). The median follow-up was 14 months (range 2-75 months).

\section{Response}

The overall response, which is a composite of complete and partial response, was $40.0 \%(16 / 40)$. In particular, $12.5 \%(5 / 40)$ of patients achieved complete response 
Fig. 2. Patient response to alitretinoin. Sixty-year-old female with MF (T2BNXM0) treated with alitretinoin $30 \mathrm{mg}$ daily. a Initial patient encounter. $\mathbf{b}$ After 8 months of treatment.
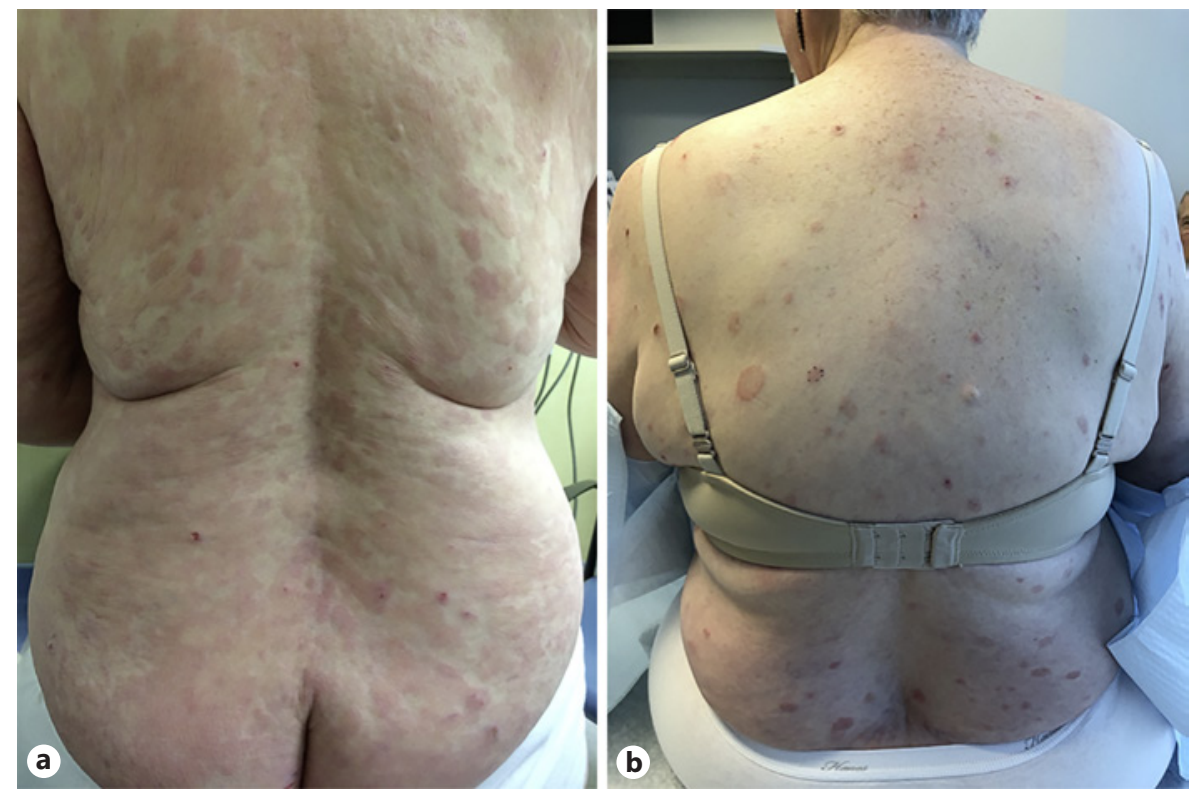

Table 4. Adverse events

Outcomes, $n /$ total (\%)
No observable adverse events
Observable adverse events
Adverse events, $n /$ total (\%)
Hypertriglyceridemia
Diarrhea
Dermatitis
Headache
Fatigue
Back pain
Discontinued, $n /$ total (\%)
Adverse events
Disease progression
Cost

$27 / 42(64.3)$

$15 / 42(35.7)$

$6 / 15(40.0)$

$2 / 15(13.3)$

$2 / 15(13.3)$

$2 / 15(13.3)$

$2 / 15(13.3)$

$1 / 15(6.7)$

$7 / 18(38.9)$

$6 / 18(33.3)$

$5 / 18(27.8)$

without any documented disease relapse (Table 3). Of those with complete response, 3 patients had early-stage MF (2 with stage IA and 1 with IB), and 2 patients had advanced-stage MF (1 with IIB and the other with SS). $27.5 \%(11 / 40)$ had a partial response with $50-99 \%$ of skin clearance (Fig. 2). 47.5\% (19/40) had stable disease, and $12.5 \%$ (5/40) developed progressive disease (Table 3). Five patients received alitretinoin monotherapy, and within this cohort, 1 patient had a complete response, another had a partial response, 2 patients had stable disease and 1 patient was lost to follow-up. When stratified by disease stage, the rate of complete response between ear-

ly-stage disease (IA-IIA) and advanced-stage disease (IIB-IVB, SS) was not significantly different $(p=1.0)$, albeit the study was not powered for this analysis.

\section{Safety and Side Effects}

The safety profile on alitretinoin was reported in 42 patients. Overall, alitretinoin was well tolerated, and $64.3 \%(27 / 42)$ patients had no reported side effects (Table 4). $35.7 \%$ (15/42) experienced at least one side effect with the most common being hypertriglyceridemia (40\%), followed by diarrhea (13.3\%), dermatitis (13.3\%), headache (13.3\%), fatigue (13.3\%) and back pain (6.4\%). There was no significant difference in the occurrence of side effects related to gender $(p=0.5)$ or disease stage $(p=0.4)$.

The rationale for alitretinoin discontinuation was reported in only 18 patients and of those patients: $38.9 \%$ (7/18) stopped due to side effects, $33.3 \%$ (6/18) discontinued due to disease progression and $27.8 \%(5 / 18)$ halted treatment due to cost (Table 4).

\section{Discussion}

When taken orally, retinoids are not considered immunosuppressive agents and therefore are an attractive option for the treatment of MF and SS [12]. Bexarotene has a response rate of approximately $50 \%$ but is not readily available in some countries including Canada $[8,9$, 13]. Based on patterns of care across Europe and the USA, 
bexarotene is one of the most frequently prescribed firstline treatments for CTCL especially in those with stage IIB disease [4]. However, the side effects associated with bexarotene are frequent, dose dependent and can lead to treatment cessation $[8,9,14]$. Other retinoids such as isotretinoin, acitretin and alitretinoin have been used off label for the management of MF with promising outcomes [6]. Therefore, consideration of other retinoids in treating MF and SS is warranted.

Like bexarotene, alitretinoin also targets the RXR but has a better safety profile, and many dermatologists have experience prescribing it for hand dermatitis and other dermatological conditions. Our retrospective study demonstrated alitretinoin is effective and relatively safe in treating MF and SS. The overall response, which is an aggregate of the complete and partial response, was $40.0 \%$ $(16 / 40)$ in our study, and there were no reported cases of disease relapse following treatment. Our response rate was lower than a previous report showing an overall response rate of $90.9 \%$ in a retrospective study of only 11 patients [10]. Such differences are reflective of the variations in sample size and the institutional differences in prescribing. However, the overall response rate of $40 \%$ for alitretinoin is comparable to that of the phase II-III studies with bexarotene, which showed an overall response of 45-55\% depending on the dose [9]. As a group, retinoids seem to be effective in treating about half of the CTCL patients, although randomized clinical trials and metaanalyses are needed to substantiate this observation.

Disease stage is an important consideration in treatment decisions for CTCL, as advanced stages can be more recalcitrant to therapy. The response to skin manifestations with bexarotene was observed across all TNM stages of CTCL [9]. In our retrospective study, 12.5\% (5/40) of patients had a complete response with 3 patients with early-stage MF and 2 patients with advanced MF, 1 with stage IIB and the other with SS. Our findings suggest that like bexarotene, alitretinoin is efficacious in treating both early and advanced stages of CTCL. However, our study was not powered to stratify response rates with alitretinoin across all CTCL stages.

Retinoids have not been formally studied in combination with other treatments for CTCL, but bexarotene has been used off label in conjunction with extracorporeal photopheresis, interferon, phototherapy or other treatments [4]. Likewise, acitretin and alitretinoin have also been combined with other therapies, commonly skin-directed therapies for the treatment of MF and SS $[10,12]$. Skin-directed therapies are used in both early stages of skin-limited disease and in later stages with systemic

Alitretinoin for the Treatment of Mycosis

Fungoides and Sézary Syndrome therapies [8]. Consistent with previous reports, $89.5 \%$ $(43 / 48)$ of patients in our study received topical corticosteroids alone or with other modalities such as narrowband ultraviolet $\mathrm{B}$ radiation (Table 2). Other therapies also used in conjunction with alitretinoin included local radiation, extracorporeal photopheresis and interferon. The need for multiple treatment modalities likely reflects the difficulty clinicians have in managing the disease and that currently no cure for CTCL exists in our repertoire of medical options.

A major drawback of bexarotene is the high rate of adverse events that can lead to treatment cessation $[8,9]$. Retinoids in general require monitoring of metabolic parameters such as triglycerides and cholesterol. In addition to these laboratory indices, bexarotene also requires close monitoring for anemia, neutropenia and hypothyroidism [9]. In contrast, alitretinoin is better tolerated than bexarotene; the most common adverse events for alitretinoin are hypertriglyceridemia, headache and cheilitis [15]. In this study, $64.3 \%(27 / 42)$ of patients had no observable side effects on alitretinoin. The most commonly observed adverse event was hypertriglyceridemia (Table 4). On average, increases in triglyceride levels can be expected in $30-44 \%$ of patients on acitretin, alitretinoin or isotretinoin, but is observed in upwards of $80 \%$ in patients on bexarotene which can precipitate pancreatitis $[6,16]$. Fibrates and $\omega-3$ fatty acids are options for lowering triglyceride levels in patients whose retinoid dosage cannot be reduced to maintain efficacy $[17,18]$. Collectively, our findings are in agreement with a previous report demonstrating that alitretinoin is well tolerated in the treatment of CTCL [10].

Being a retrospective analysis, this study has several limitations. First, although the same data collection template was used across the study sites, there were some inconsistencies with the cohort and reporting of data. Second, only $10.4 \%(5 / 48)$ of patients received alitretinoin monotherapy, whereas most patients received alitretinoin in combination with standard treatments. Of those on alitretinoin alone, $60 \%(3 / 5)$ had a complete or partial response, 1 had stable disease and 1 was lost to follow-up. However, one third of patients received only topical corticosteroids with alitretinoin. Combination therapies are routinely utilized to treat CTCL, which reflects the complexity of a disease that requires multiple treatment modalities. Third, this retrospective analysis included patients with early and advanced stages of the disease, but our study was not powered to assess the efficacy of alitretinoin across the different stages of CTCL. The studies with bexarotene did not report efficacy stratified by dis- 
ease stage either $[8,9]$. We were also unable to comment on metabolic and laboratory monitoring parameters for patients on alitretinoin and to describe the adverse events in accordance with the common terminology criteria for adverse events. Overall, our retrospective review is in agreement with previous reports that alitretinoin is effective in the treatment of MF and SS, with few adverse events. Additional prospective and randomized studies with alitretinoin are needed to validate these observations.

\section{Conclusions}

Although bexarotene is used in Europe for the treatment of CTCL, it is not readily available in some countries such as Canada and has significant side effects that can lead to treatment cessation. Alitretinoin is effective and safe in the treatment of MF and SS and limits disease progression alone or in combination with standard therapies for early and advanced CTCL. Given its lower rate of side effects, alitretinoin appears to be a viable alternative in treating MF and SS.

\section{Key Message}

Alitretinoin is effective and safe in the treatment of mycosis fungoides and Sézary syndrome.

\section{Acknowledgments}

We would like to thank Dr. Ivan Litinov and Dr. Gilles Lauzon for their assistance with the study design.

\section{Statement of Ethics}

The patients in this paper provided written informed consent to publication of their case details. The study protocols were approved by the respective institute's committee on human research.

\section{Conflict of Interest Statement}

The authors have no conflicts of interest.

\section{Funding Sources}

Dr. Iris Wohlmuth-Wieser is supported by the Austrian Science Fund (FWF J 4382-B).

\section{Author Contributions}

T.T.V., R.A., R.G. and J.H. contributed to the development of the study protocol and were involved in drafting the manuscript or revising it for important intellectual content. N.A. and I.W.-W. analyzed and interpreted the data. L.S., J.-D.M.H. and J.C. assisted with protocol design, collected and analyzed data. S.W. and N.S. were involved in critical review of the paper and contributing to intellectual content.

\section{References}

1 Wilcox RA. Cutaneous T-cell lymphoma: 2017 update on diagnosis, risk-stratification, and management. Am J Hematol. 2017 Oct; 92(10):1085-102.

2 Girardi M, Heald PW, Wilson LD. The pathogenesis of mycosis fungoides. N Engl J Med. 2004 May;350(19):1978-88.

3 Mourad A, Gniadecki R. Overall Survival in Mycosis Fungoides: A Systematic Review and Meta-Analysis. J Invest Dermatol. 2020 Feb; 140(2):495-497.e5.

4 Quaglino P, Maule M, Prince HM, Porcu P, Horwitz S, Duvic M, et al. Global patterns of care in advanced stage mycosis fungoides/ Sezary syndrome: a multicenter retrospective follow-up study from the Cutaneous Lymphoma International Consortium. Ann Oncol. 2019 Mar;30(3):494.

5 Sessler RJ, Noy N. A ligand-activated nuclear localization signal in cellular retinoic acid binding protein-II. Mol Cell. 2005 Apr;18(3): $343-53$.
6 Huen AO, Kim EJ. The Role of Systemic Retinoids in the Treatment of Cutaneous T-Cell Lymphoma. Dermatol Clin. 2015 Oct;33(4): 715-29.

7 Khalil S, Bardawil T, Stephan C, Darwiche N, Abbas O, Kibbi AG, et al. Retinoids: a journey from the molecular structures and mechanisms of action to clinical uses in dermatology and adverse effects. J Dermatolog Treat. 2017 Dec;28(8):684-96.

8 Duvic M, Martin AG, Kim Y, Olsen E, Wood GS, Crowley CA, et al.; Worldwide Bexarotene Study Group. Phase 2 and 3 clinical trial of oral bexarotene (Targretin capsules) for the treatment of refractory or persistent earlystage cutaneous T-cell lymphoma. Arch Dermatol. 2001 May;137(5):581-93.

9 Duvic M, Hymes K, Heald P, Breneman D, Martin AG, Myskowski P, et al.; Bexarotene Worldwide Study Group. Bexarotene is effective and safe for treatment of refractory advanced-stage cutaneous $\mathrm{T}$-cell lymphoma: multinational phase II-III trial results. J Clin Oncol. 2001 May;19(9):2456-71.
10 Kapser C, Herzinger T, Ruzicka T, Flaig M, Molin S. Treatment of cutaneous T-cell lymphoma with oral alitretinoin. J Eur Acad Dermatol Venereol. 2015 Apr;29(4):783-8.

11 Olsen EA, Whittaker S, Kim YH, Duvic M, Prince HM, Lessin SR, et al.; International Society for Cutaneous Lymphomas; United States Cutaneous Lymphoma Consortium; Cutaneous Lymphoma Task Force of the European Organisation for Research and Treatment of Cancer. Clinical end points and response criteria in mycosis fungoides and Sézary syndrome: a consensus statement of the International Society for Cutaneous Lymphomas, the United States Cutaneous Lymphoma Consortium, and the Cutaneous Lymphoma Task Force of the European Organisation for Research and Treatment of Cancer. J Clin Oncol. 2011 Jun;29(18):2598-607.

12 Cheeley J, Sahn RE, DeLong LK, Parker SR. Acitretin for the treatment of cutaneous Tcell lymphoma. J Am Acad Dermatol. 2013 Feb;68(2):247-54.

Alhusayen et al. 
13 Panchal MR, Scarisbrick JJ. The utility of bexarotene in mycosis fungoides and Sézary syndrome. Onco Targets Ther. 2015 Feb;8:36773.

14 Talpur R, Ward S, Apisarnthanarax N, Breuer-Mcham J, Duvic M. Optimizing bexarotene therapy for cutaneous T-cell lymphoma. J Am Acad Dermatol. 2002 Nov;47(5): 672-84.

15 Ruzicka T, Larsen FG, Galewicz D, Horváth A, Coenraads PJ, Thestrup-Pedersen K, et al. Oral alitretinoin (9-cis-retinoic acid) therapy for chronic hand dermatitis in patients refractory to standard therapy: results of a random- ized, double-blind, placebo-controlled, multicenter trial. Arch Dermatol. 2004 Dec; 140(12):1453-9.

16 Klör HU, Weizel A, Augustin M, Diepgen TL, Elsner P, Homey B, et al. The impact of oral vitamin A derivatives on lipid metabolism What recommendations can be derived for dealing with this issue in the daily dermatological practice? J Dtsch Dermatol Ges. 2011 Aug;9(8):600-6.

17 Musolino A, Panebianco M, Zendri E, Santini M, Di Nuzzo S, Ardizzoni A. Hypertriglyceridaemia with bexarotene in cutaneous $\mathrm{T}$ cell lymphoma: the role of omega-3 fatty acids. Br J Haematol. 2009 Apr; 145(1): 84-6.

18 Skulas-Ray AC, Wilson PW, Harris WS, Brinton EA, Kris-Etherton PM, Richter CK, et al.; American Heart Association Council on Arteriosclerosis, Thrombosis and Vascular Biology; Council on Lifestyle and Cardiometabolic Health; Council on Cardiovascular Disease in the Young; Council on Cardiovascular and Stroke Nursing; and Council on Clinical Cardiology. Omega-3 Fatty Acids for the Management of Hypertriglyceridemia: A Science Advisory From the American Heart Association. Circulation. 2019 Sep;140(12):e673-91. 